\title{
Quantum transport in ferromagnetic permalloy nanostructures
}

\author{
D. Neumaier, * A. Vogl, J. Eroms, and D. Weiss \\ Institut für Experimentelle und Angewandte Physik, Universität Regensburg, 93040 Regensburg, Germany \\ (Received 1 September 2008; revised manuscript received 13 October 2008; published 24 November 2008)
}

\begin{abstract}
We studied phase-coherent phenomena in mesoscopic permalloy $\left(\mathrm{Ni}_{81} \mathrm{Fe}_{19}\right)$ samples by exploring lowtemperature transport. Both differential conductance as a function of bias voltage and magnetoconductance of individual wires display conductance fluctuations. Analysis of these fluctuations yields a phase coherence length of $\sim 250 \mathrm{~nm}$ at $25 \mathrm{mK}$ as well as a $1 / \sqrt{T}$ temperature dependence. To suppress conductance fluctuations by ensemble averaging, we investigated low-temperature transport in wire arrays and extended permalloy films. In these samples we have measured conductance corrections which stem from electron-electron interaction; but attempts to detect signatures of weak localization were without success.
\end{abstract}

DOI: 10.1103/PhysRevB.78.174424

PACS number(s): 73.63.-b, 73.23.-b, 73.20.Fz

\section{INTRODUCTION}

In nanoscale samples the conductance is affected by quantum interference effects at sufficiently low temperatures, including weak localization (WL), ${ }^{1}$ universal conductance fluctuations (UCF), ${ }^{2}$ or Aharonov-Bohm oscillations. ${ }^{3}$ All these effects rely on the electron's wave nature and require phase-coherent transport over a certain distance. In nonmagnetic metals interference effects have been investigated intensely over the last two decades (for a review see, e.g., Ref. 4), showing that the electrons can propagate up to several microns without losing their phase information although their mean-free path is much smaller. For ferromagnetic metals, in contrast, only a few experimental works on phasecoherent phenomena exist (e.g., Refs. 5-7). While AharonovBohm oscillations or universal conductance fluctuations have been observed in permalloy ${ }^{6}$ and cobalt devices, ${ }^{5}$ the suppression of weak localization is still an open question. Up to now no clear signature of weak localization was found in any ferromagnetic metal. For example, the conductances of $\mathrm{Co},{ }^{5,8}$ $\mathrm{Co} / \mathrm{Pt}$ multilayers, ${ }^{9} \mathrm{Fe},{ }^{10}$ and Ni (Ref. 11) were not affected by weak localization. In these materials a decreasing conductance with decreasing temperature was ascribed to electronelectron interaction. ${ }^{5,8-11}$ However, in the ferromagnetic semiconductor (Ga,Mn)As (Ref. 12) weak localization corrections could be observed quite recently. ${ }^{13,14}$ In contrast to ferromagnetic metals the internal magnetic induction of $(\mathrm{Ga}, \mathrm{Mn}) \mathrm{As}$ is rather small. In this work we will investigate universal conductance fluctuations, weak localization, and electron-electron interaction in permalloy nanostructures.

\section{SAMPLE PREPARATION AND MEASUREMENT TECHNIQUE}

For the experiments we fabricated single wires, arrays of wires connected in parallel, and thin film areas using electron-beam lithography (a Zeiss electron microscope equipped with a nanonic pattern generator) and thermal evaporation of permalloy $\left(\mathrm{Ni}_{81} \mathrm{Fe}_{19}\right)$. The contacts to the samples were made out of gold after a brief in situ ion-beam etching to remove the oxide. An electron micrograph of a 145-nm-long, 35-nm-wide, and 15-nm-thick permalloy wire is shown in Fig. 1(a).
All measurements have been performed in a top-loading dilution refrigerator using standard four-probe lock-in techniques. The external magnetic field was always applied perpendicular to the plane. To avoid heating of the electrons careful shielding as well as small measuring currents (depending on the sample's resistance ranging from 4 to 400 nA) were crucial. To measure the differential resistance we also used standard lock-in techniques. A dc voltage $U_{\mathrm{dc}}$ was superimposed upon a comparably small ac voltage $U_{\mathrm{ac}}$ $(5 \mu \mathrm{V}$ ) having a frequency of $417 \mathrm{~Hz}$. The conductance $G$ was obtained by inverting the resistance $R$ of the samples $G=1 / R$, while the differential conductance was obtain by inverting the differential resistance $G^{d}=1 / R^{d}=1 /(d U / d I)$.

\section{UNIVERSAL CONDUCTANCE FLUCTUATIONS IN SINGLE WIRES}

UCF result from correlations between different transmission paths through a disordered mesoscopic sample (Ref. 15 and references therein). Hence the conductance $G$ of a mesoscopic sample is sensitive to the impurity configuration. Changing the applied magnetic field $B$ or the applied bias voltage $U$ leads to aperiodic conductance fluctuations. ${ }^{15}$ While changing in the magnetic field leads to a relative phase shift of different paths due to the Aharonov-Bohmeffect, a change in bias voltage changes the electron's energy and thus the corresponding wavelength. For observing UCF, phase coherence is necessary. If the sample is larger than the phase coherence length in one or more spatial dimensions, the fluctuations get damped until the classical value of conductance is reached. ${ }^{2}$ Hence sufficiently small samples and low temperatures are required to observe universal conductance fluctuations.

Here we investigate conductance fluctuations in differential conductance $G^{d}(U)$ and in magnetoconductance $G(B)$ of two single Py wires having a length of 145 and $330 \mathrm{~nm}$, respectively. The width of both wires is $35 \mathrm{~nm}$ and the thickness is $15 \mathrm{~nm}$. An electron micrograph of the 145-nm-long wire is shown in Fig. 1(a).

The differential conductance $G^{d}\left(U_{\mathrm{dc}}\right)$ as a function of the applied bias voltage $U_{\mathrm{dc}}$ of the 145-nm-long wire is shown in Fig. 1(b) for temperatures $T$ ranging from 120 to $800 \mathrm{mK}$. The magnetic field applied normal to the sample was $3 \mathrm{~T}$ 
a)

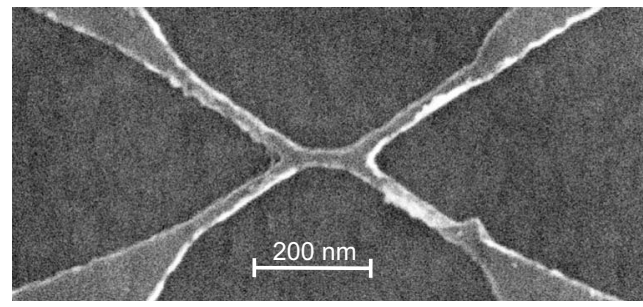

b)
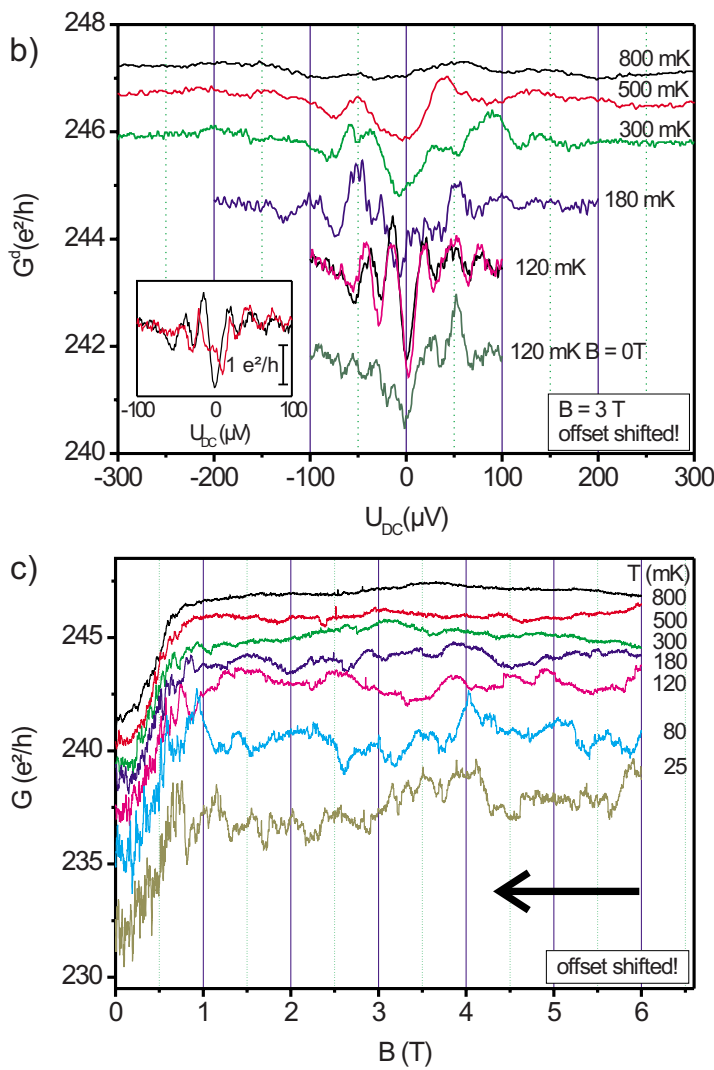

FIG. 1. (Color online) (a) Electron micrograph of the 145-nmlong permalloy wire. (b) Differential conductance of the 145-nmlong permalloy wire measured at different temperatures. The applied magnetic field was $3 \mathrm{~T}$ (except for the lowest trace, here $B$ $=0$ ). The different traces, except the $800 \mathrm{mK}$ trace, were shifted for clarity. The reproducibility is shown for the $120 \mathrm{mK}$ trace (purple and black trace). The inset shows the differential conductance at $120 \mathrm{mK}$ and $3 \mathrm{~T}$ before and after the field was raised to $4 \mathrm{~T}$. The black trace in the inset corresponds to the black trace at $120 \mathrm{mK}$ in the main figure. (c) Magnetoconductance of the 145-nm-long wire in a perpendicular applied magnetic field at different temperatures. Also here, the different traces, except the $800 \mathrm{mK}$ trace, were shifted for clarity. The arrow gives the sweeping direction.

(except for the lowest trace, here $B=0$ ). At all temperatures fluctuations in the differential conductance are visible, showing that phase-coherent transport takes place in the wire. The reproducibility of the differential conductance is demonstrated for the $T=120 \mathrm{mK}$ trace. With increasing temperature the fluctuation amplitude gets reduced. This shows that the sample is larger than the phase coherence length at least at $180 \mathrm{mK}$. Also with increasing dc voltage the amplitude of the fluctuations decreases. This suggests that the applied voltage causes heating. The correlation voltage $U_{C}$ the av-

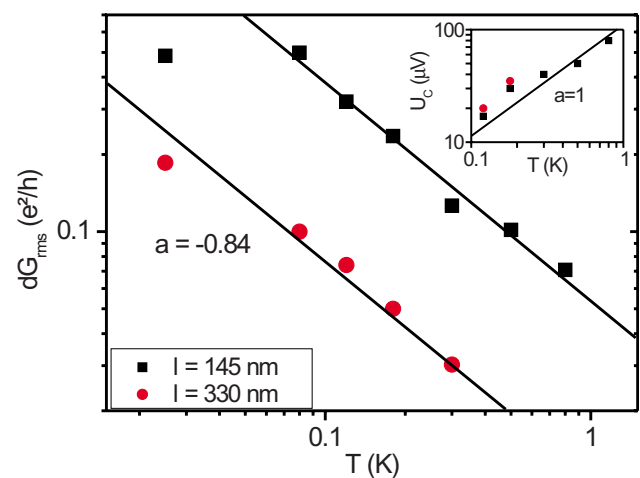

FIG. 2. (Color online) Amplitude of conductance fluctuations $d G_{\text {rms }}$ measured in the magnetoconductance of the 145-nm-long (black squares) and 330-nm-long wire (red circles). The lines are a guide for the eyes and give a slope of $a=-0.84$. The inset shows the correlation voltage of both wires for different temperatures. Also here black squares correspond to the 145-nm-long wire and red circles to the 330-nm-long wire. The straight line is a guide for the eyes and gives a slope of $a=1$.

erage spacing between maxima and minima-gives the energy, which is necessary to change the electron's phase by $\pi$. Here $U_{C}$ increases with increasing temperature. The correlation voltage is related to the dephasing time $\tau_{\phi}$ by $|e| U_{C}$ $=\hbar / \tau_{\phi} \cdot{ }^{16}$ Hence the dephasing time decreases with increasing temperature as expected. ${ }^{17}$ At $T=120 \mathrm{mK}$ the correlation voltage is approximately $17 \mu \mathrm{V}$. This corresponds to a dephasing time of $40 \mathrm{ps}$. With the diffusion constant of permalloy $D \approx 4 \times 10^{-4} \mathrm{~m}^{2} / \mathrm{V} \mathrm{s},{ }^{18}$ we obtain for the phase coherence length $L_{\phi}=\sqrt{D \tau_{\phi}}=130 \mathrm{~nm}$ at $120 \mathrm{mK}$. For comparison, the thermal energy $k_{B} T=10 \mu \mathrm{eV}$ at $120 \mathrm{mK}$. Hence thermal broadening does not lead to a suppression of interference as $k_{B} T<e U_{C} \cdot{ }^{19}$

To investigate the temperature dependency of the dephasing time we plotted the correlation voltage of the $145-\mathrm{nm}$ long wire in a log-log diagram versus temperature (see inset of Fig. 2). The slope of the correlation voltage can be approximated by 1 . This corresponds to a dephasing time $\tau_{\phi}$ $\propto 1 / T$ and thus to a phase coherence length $L_{\phi} \propto 1 / \sqrt{T}$. We note that the estimate of the correlation voltage is associated with a relatively high degree of uncertainty, as only the region around zero voltage can be used for an estimate of $U_{C}$. Hence the slope gives only a rough estimate of the temperature dependency.

The differential conductance of the 330-nm-long wire also exhibits reproducible fluctuations associated with phasecoherent transport (not shown). At $T=120 \mathrm{mK}$ the correlation voltage in the $330-\mathrm{nm}$-long wire is $20 \mu \mathrm{V}$, which is quite close to the correlation voltage observed in the 145$\mathrm{nm}$-long wire at the same temperature. Hence the dephasing time and the phase coherence length are quite similar in both wires, as the measurement of the correlation voltage gives the dephasing time independent of the sample's geometry. In the 330-nm-long wire the temperature dependence of the correlation voltage could not be estimated properly as the fluctuations disappear above $180 \mathrm{mK}$. This is due to the damping of the conductance fluctuations with increasing sample size. The correlation voltage taken at $T=120$ and $180 \mathrm{mK}$ gives a 
temperature dependency in good agreement with the one obtained in the 145-nm-long wire (see inset of Fig. 2).

In mesoscopic samples reproducible conductance fluctuations are also visible in the magnetoconductance $G(B)$, also known as magnetic fingerprint of the sample. ${ }^{2}$ In Fig. 1(c) the magnetoconductance of the 145-nm-long wire is shown for temperatures ranging from 25 to $800 \mathrm{mK}$, exhibiting conductance fluctuations vanishing with increasing temperature. The positive magnetoconductance at $B=0 \ldots 0.7 \mathrm{~T}$ is due to the anisotropic magnetoresistance (AMR) in ferromagnets. ${ }^{20}$ For a magnetization aligned in wire direction (here the easy magnetic axis) the conductance is smaller than for perpendicular orientation (here a hard magnetic axis). In contrast to the fluctuations of the differential conductance, the magnetoconductance fluctuations are not reproducible within several sweeps. As the conductance fluctuations only appear at low temperatures and decrease with increasing temperature and increasing wire length, we ascribe them to phase-coherent phenomena. To check whether the fluctuations originate from time-dependent fluctuations, we measured the conductance at a fixed magnetic field for the time interval of a magneticfield sweep. Time-dependent fluctuations are visible, but their amplitude is by approximately a factor of 4 , lower compared to the magnetoconductance fluctuations. Additionally, the time scale of fluctuations is longer in the time sweep compared to the magnetic-field sweep. Hence we can conclude that the observed conductance fluctuations, shown in Fig. 1(c), are primarily due to the changes in $B$ but only superimposed by time-depending fluctuations. Timedependent universal conductance fluctuations in permalloy nanowires are in the focus of Ref. 7. A possible explanation for irreproducible magnetoconductance traces could be a change in the scatterer configuration due to magnetostriction. While the fluctuations in the differential conductance are well reproducible for a fixed magnetic field [see Fig. 1(b)], they get less reproducible when the magnetic field is changed between the measurements. This is shown in Fig. 1(b). The two traces in the inset show the differential conductance at $T=120 \mathrm{mK}$ and $B=3 \mathrm{~T}$. Between the measurements the magnetic field was increased to $4 \mathrm{~T}$ and then reduced to $3 \mathrm{~T}$ again (the black trace shows the differential conductance at the beginning and the red trace after the magnetic field was varied). This magnetic-field change reduces the reproducibility of the traces compared to the two traces shown in Fig. 1 (b) at $120 \mathrm{mK}$ (black and purple), where the magnetic field was not changed between the measurements. Hence, changing the magnetic field seems to change, to some extent, the impurity configuration and hence the pattern of the conductance fluctuation as a function of $B$. Magnetostriction although weak in Py might be a possible reason for the observed irreproducibility.

In the low-field region $(|B|<0.7 \mathrm{~T})$, the region where the magnetization rotates from in plane to perpendicular to the plane, the conductance fluctuations are more pronounced than in the high-field region. Such a behavior has already been observed in (Ga,Mn)As wires ${ }^{21,22}$ and ad hoc ascribed to the formation of domain walls. ${ }^{22}$ Analyzing the fluctuations in the differential conductance at zero magnetic field [Fig. 1(b), lowest trace] one finds that neither the amplitude nor the correlation voltage is significantly different compared to $B=3 \mathrm{~T}$. This implies that the dephasing time is not much affected due to the presence of domain walls in the low-field region. A similar result was found in Ref. 7 analyzing timedependent universal conductance fluctuations (TDUCFs) in permalloy nanowires. Their analysis of TDUCFs supports the idea that domain walls act as coherent scatterers. ${ }^{7}$ In the following the analysis is limited to high magnetic fields $(B$ $=1 \ldots 6 \mathrm{~T}$ ) where the magnetization is saturated perpendicular to the plane.

The root-mean-square (rms) amplitude of the conductance fluctuations $d G_{\mathrm{rms}}$ of the 145-nm-long and 330-nm-long wires (taken at $B=1 \ldots 6 \mathrm{~T}$ ) is plotted in a log-log diagram versus $T$ in Fig. 2. Here $d G_{\mathrm{rms}}=\sqrt{\left\langle(G-\langle G\rangle)^{2}\right\rangle}$, where $\langle\ldots\rangle$ denotes the averaging over $B$. For a quasi-one-dimensional (1D) wire $\left(w, t<L_{\phi}<l\right)$ one finds for the amplitude of the conductance fluctuations, ${ }^{2}$

$$
d G_{\mathrm{rms}}=C \frac{e^{2}}{h}\left(\frac{L_{\phi}}{l}\right)^{3 / 2} .
$$

Here, $l$ is the wire length and $C$ is a constant, with a value close to or smaller than unity depending, e.g., on the strength of spin-orbit coupling ${ }^{23}$ and the applied magnetic field. ${ }^{2}$ Equation (1) is applicable to describe the conductance fluctuations as long as thermal averaging can be ruled out: $L_{\phi}$ $<L_{T}=\sqrt{\hbar D / k_{B} T}$, which is equivalent to $e U_{C}>k_{B} T$. In our permalloy wire this is the case as shown above, and Eq. (1) can be used to extract the phase coherence length. $d G_{\mathrm{rms}}$ of the 145-nm-long wire is increasing with decreasing temperature until it starts to saturate at $80 \mathrm{mK}$ at a value of $0.5 e^{2} / h$ (see Fig. 2). For such a saturation, there are essentially three possible reasons:

(1) The effective electron temperature is higher than the bath temperature due to a too high measuring current or external rf noise.

(2) Magnetic impurities lead to a saturation of $L_{\phi}$ due to Kondo scattering as observed in normal metals. ${ }^{24}$

(3) The phase coherence length reaches the wire length and the wire is no longer quasi-one-dimensional. In that case $d G_{\mathrm{rms}}=C e^{2} / h^{2}$

As we deal here with a ferromagnet and since saturation is only observed in the shorter one of the two wires, an intrinsic saturation of the phase coherence length due to Kondo scattering is unlikely. While the amplitude of the conductance fluctuations of the 25 and $80 \mathrm{mK}$ trace in Fig. 1(c) is the same, the correlation field $B_{C}$ is smaller at $25 \mathrm{mK}$. The correlation field defines the typical field scale on which the conductance fluctuates and is related to the maximum area enclosed by a phase-coherent trajectory. In one-dimensional samples $B_{C}=\frac{h}{e} \frac{1}{w L_{\phi}} \cdot{ }^{2}$ We note that the correlation field might not be a well-defined quantity here, as the impurity configuration might be changed by magnetostriction, as discussed above. Hence the correlation field can only serve as a very rough estimate of the phase coherence length. At $80 \mathrm{mK}$ the correlation field is between $\sim 0.6$ and $\sim 0.8 \mathrm{~T}$. This corresponds to a phase coherence length of $\sim 150-200 \mathrm{~nm}$. When extrapolating the correlation voltage $U_{C}$ (see inset of Fig. 2) down to $80 \mathrm{mK}$, one arrives at a phase coherence length of $160 \mathrm{~nm}$. This is in very good agreement with the 
phase coherence length estimated from the correlation field. Hence, the saturation of $d G_{\mathrm{rms}}$ observed in the 145-nm-long wire below $80 \mathrm{mK}$ is most likely due to a dimensional crossover from quasi $1 \mathrm{D}$ to $0 \mathrm{D}$ and the saturation value of $d G_{\mathrm{rms}}$ is $\sim 0.5 e^{2} / h$.

In both wires the temperature dependency of $d G_{\mathrm{rms}}$ can be approximated by a power law, $d G_{\mathrm{rms}} \propto T^{-0.84}$. Using Eq. (1), the temperature dependency of the dephasing length is $L_{\phi}$ $\propto T^{-0.55}$. This temperature dependency agrees well with the one estimated using the correlation voltage $U_{C}\left(L_{\phi} \propto T^{-0.5}\right)$. In permalloy wires noise measurements reveal a temperature dependency of $L_{\phi}$ probably steeper than $T^{-0.57}$, but the analysis was complicated by several uncertainties. Also the wires investigated in Ref. 7 were quasi-two-dimensional (2D) because of the higher temperature $(T>2 \mathrm{~K})$. Hence a direct comparison is difficult. In ferromagnetic $(\mathrm{Ga}, \mathrm{Mn}) \mathrm{As}$ wires the phase coherence length followed a $T^{-0.5}$ dependency $^{21,22}$ and was associated with critical electron-electron scattering (CEEI). ${ }^{21,25,26}$ For permalloy CEEI seems not to be a suitable candidate for dephasing as CEEI describes dephasing in a strongly disordered metal near the metal-insulator transition (MIT). In metals far away from the MIT dephasing is usually ascribed to Nyquist scattering leading to a phase coherence length $\propto T^{-1 / 3}$ in $1 \mathrm{D}$ systems. ${ }^{27}$ So, the microscopic mechanism of dephasing in permalloy remains an open issue.

The value of the phase coherence length at $25 \mathrm{mK}$ is approximately $250 \mathrm{~nm}$. We arrive at this value by analyzing the magnetoconductance fluctuations of the 330-nm-long wire $\left(L_{\phi}=180 \mathrm{~nm}\right)$ or by extrapolating the magnetoconductance fluctuations of the 145-nm-long wire down to $25 \mathrm{mK}$ $\left(L_{\phi}=260 \mathrm{~nm}\right)$. We note that there is some uncertainty in determining the length of the wires, especially in the region of the voltage probes where the wire widens up. This uncertainty enters the phase coherence length linearly. Taking the correlation energy and extrapolating the value of $U_{C}$ down to $25 \mathrm{mK}$ leads to a phase coherence length of $\sim 260 \mathrm{~nm}$ (145$\mathrm{nm}$-long wire) and $\sim 240 \mathrm{~nm}$ (330-nm-long wire). This approximation is independent of the exact wire geometry. In Ref. 6 the phase coherence length in permalloy was extracted from periodic Aharonov-Bohm oscillations. Their value of $L_{\phi}=500 \mathrm{~nm}$ taken at $30 \mathrm{mK}$ is by a factor of 2 larger than the value estimated here. The difference might be explained by different material composition although the nominal composition in Ref. 6 was specified to be $\mathrm{Ni}_{81} \mathrm{Fe}_{19}$, the same one as used here. The saturation field of the magnetization observed in a perpendicular external field defined by the saturation field of the AMR is in Ref. $6(\sim 1.2 \mathrm{~T})$. For permalloy $\left(\mathrm{Ni}_{81} \mathrm{Fe}_{19}\right)$ the saturation magnetization is $1.0 \mathrm{~T}$. Hence the saturation field is $1.0 \mathrm{~T}$ in an extended film and $0.5 \mathrm{~T}$ in a cigar-shaped ellipsoid with infinite aspect ratio (comparable to a long wire with a very small cross section) and with the long axis perpendicular to the external field. ${ }^{28}$ For the geometry used in Ref. 6 (a 2.5- $\mu$ m-long wire with a cross section of $40 \times 20 \mathrm{~nm}^{2}$ having a ring of $500 \mathrm{~nm}$ diameter and the same cross section in the middle; see Ref. 6 for details) one would expect a saturation field above but still close to $0.5 \mathrm{~T}$. In our samples the saturation field is $\sim 0.7 \mathrm{~T}$ in the $145-\mathrm{nm}-$ long and 330-nm-long wires [see Fig. 1(c)], $\sim 1.0 \mathrm{~T}$ in the extended film [see inset of Fig. 3(a)], and $\sim 0.6 \mathrm{~T}$ in the wire array [see inset of Fig. 3(b)]. These values are consistent
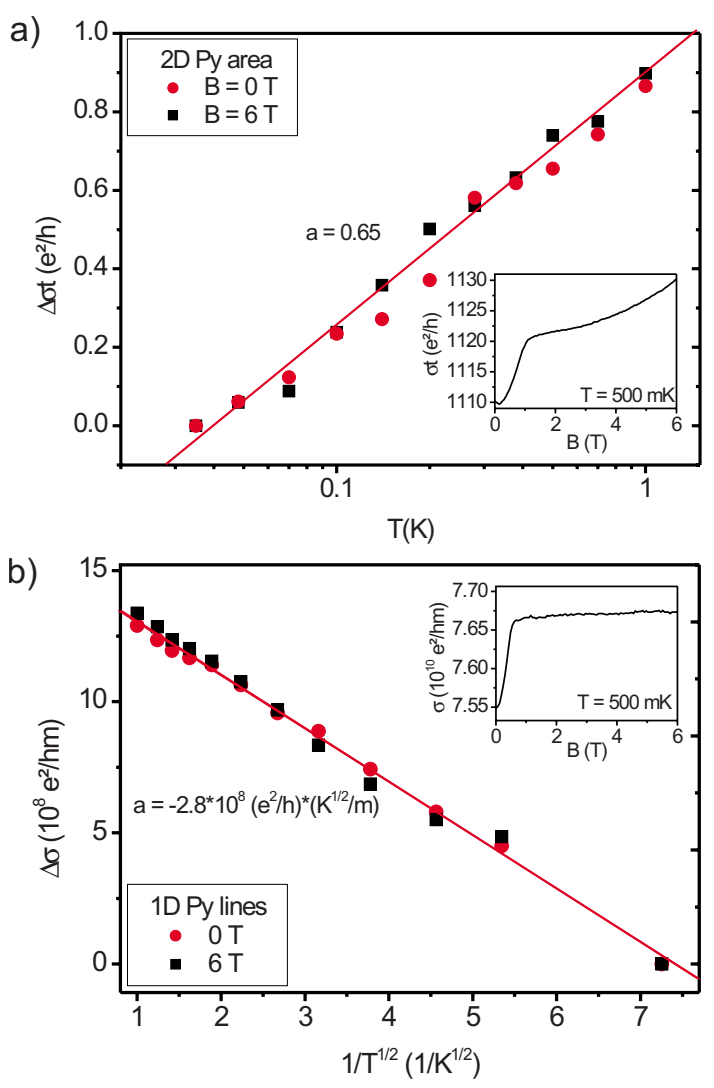

FIG. 3. (Color online) (a) Change in conductivity $\Delta \sigma$ multiplied with the layer thickness $t$ of the two-dimensional square at zero field (red circles) and $B=6 \mathrm{~T}$ (black squares) plotted versus $\log T$. The line is the best linear fit of the $6 \mathrm{~T}$ data and has a slope of 0.65 . The inset shows the magnetoconductance at $T=500 \mathrm{mK}$. (b) Conductivity change in a quasi-one-dimensional line array at zero field (red circles) and $B=6 \mathrm{~T}$ (black squares) plotted versus $1 / \sqrt{T}$. The line is the best linear fit of the $6 \mathrm{~T}$ data and has a slope of -2.8 $\times 10^{8}\left(e^{2} / h\right)(\sqrt{\mathrm{K}} / \mathrm{m})$. The inset shows the magnetoconductance at $T=500 \mathrm{mK}$.

with the ones expected for permalloy. Hence the material used in Ref. 6 seems to be quite different from the material used here, and the values of the phase coherence length can hardly be compared.

\section{ELECTRON-ELECTRON INTERACTION IN 1D AND 2D SAMPLES}

At low temperatures quantum interference effects such as WL (Ref. 1) or electron-electron interaction (EEI) (Ref. 29) lead to a reduction in the conductance even in macroscopic samples. The effect of weak localization originates from the constructive interference of partial waves traveling on timereversed paths. This leads to an increased probability of backscattering and hence reduces the conductance. As a magnetic field removes time-reversal symmetry, weak localization gets suppressed in the presence of a magnetic field and the corresponding magnetoconductance is positive. ${ }^{1}$ In permalloy wires and films a positive magnetoconductance in a perpendicular magnetic field, as expected for WL, is already present due to the AMR. ${ }^{20}$ But in contrast to WL the 
AMR is independent of the temperature, at least below $1 \mathrm{~K}$, and thus does not lead to a change in conductivity with decreasing temperature. Electron-electron interaction, on the other hand, arises from a modified screening of the Coulomb potential due to the diffusive propagation. ${ }^{29}$ The size and the temperature dependencies of the conductivity correction are very similar for EEI and WL at zero field $;{ }^{29}$ but in contrast to WL, EEI is not suppressed by a magnetic field. Hence the different effects (AMR, WL, and EEI) can be distinguished quite effectively by comparing the conductivity decrease at different magnetic fields. In case of WL the conductivity at zero field is expected to decrease more precipitously with decreasing $T$ compared to the case with finite $B$.

As ferromagnets have an internal magnetic induction, the question arises whether WL can be observed in a ferromagnet at all. Up to now several experimental works explore this question showing that $\mathrm{WL}$ is absent in $\mathrm{Co},{ }^{8,10} \mathrm{Fe},{ }^{10} \mathrm{Ni},{ }^{11}$ and $\mathrm{Co} / \mathrm{Pt}$ multilayers. ${ }^{9}$ In the ferromagnetic semiconductor $(\mathrm{Ga}, \mathrm{Mn})$ As, having a rather small internal magnetic induction, weak localization was observed. ${ }^{13,14}$ As theory claims that WL does not get suppressed by internal magnetic induction, ${ }^{30,31}$ the question arises why WL can be observed in $(\mathrm{Ga}, \mathrm{Mn}) \mathrm{As}$ but not in $\mathrm{Co}, \mathrm{Fe}, \mathrm{Ni}$, or $\mathrm{Co} / \mathrm{Pt}$ multilayers. One important difference between these materials is the phase coherence length. While $L_{\phi} \approx 160 \mathrm{~nm}$ at $20 \mathrm{mK}$ is quite large in $(\mathrm{Ga}, \mathrm{Mn}) \mathrm{As},{ }^{25}$ the phase coherence length is comparably small in Co $\left[L_{\phi} \approx 30 \mathrm{~nm}\right.$ at $30 \mathrm{mK}$ (Ref. 5) $]$ and $\mathrm{Ni}\left[L_{\phi} \approx 80 \mathrm{~nm}\right.$ at $30 \mathrm{mK}$ (Ref. 18)]. Hence permalloy is a quite promising candidate for exploring $\mathrm{WL}$ as it combines a strong internal magnetic induction $\left[H_{\mathrm{Int}}=1.0 \mathrm{~T}\right.$ compared to $40 \mathrm{mT}$ in $(\mathrm{Ga}, \mathrm{Mn}) \mathrm{As}$ (Ref. 12)] with a relatively large phase coherence length of approximately $250 \mathrm{~nm}$ at $25 \mathrm{mK}$.

To study permalloy for WL/EEI experiments, we fabricated an extended square $(w=l=4 \mu \mathrm{m}$ and $t=15 \mathrm{~nm})$ and an array of six wires in parallel $(w=20 \mathrm{~nm}, l=4 \mu \mathrm{m}$, and $t=15 \mathrm{~nm})$ and measured their conductivity at different temperatures with and without an applied perpendicular magnetic field. The square is expected to behave as quasi-twodimensional, as $w, l>L_{\phi}, L_{T}>t$, and the wire array is expected to behave as quasi-one-dimensional, as $l>L_{\phi}, L_{T}$ $>w, t$ at temperature below $1 \mathrm{~K}$. The magnetoconductance of the square and the wire array are shown in the insets of Fig. 3 for $T=500 \mathrm{mK}$. Due to ensemble averaging universal conductance fluctuations are suppressed in both systems. For both samples the AMR is visible up to 1 (area) and $0.6 \mathrm{~T}$ (wires) as expected for permalloy and the corresponding saturation fields. ${ }^{28}$ The temperature dependency of the conductivity change in the area, relative to $T=35 \mathrm{mK}$, is shown in Fig. 3(a) for $B=6 \mathrm{~T}$ (black squares) and zero field (red circles). At both magnetic fields the conductivity follows a logarithmical temperature dependency with a slope independent of the applied magnetic field. Hence WL can be ruled out as origin of the conductivity decrease, as WL gets suppressed by an external field. EEI interaction leads to a conductivity decrease independent of the applied magnetic field. For the conductivity decrease due to EEI one obtains in twodimensional films, ${ }^{29}$

$$
\Delta \sigma t=\frac{F^{2 \mathrm{D}}}{\pi} \frac{e^{2}}{h} \log \frac{T}{T_{0}},
$$

with the parameter $F^{2 \mathrm{D}}=2.3(1-0.75 \widetilde{F})$, where $\tilde{F}$ is a factor depending on the dimensionality and the reduction in the Hartree term for a static-screened interaction. ${ }^{29}$ In our permalloy square one obtains a parameter $F^{2 \mathrm{D}}=2.0$, using a slope of 0.65 as shown in Fig. 3(a). This value of $F^{2 \mathrm{D}}$ is in excellent agreement with the parameters found in other ferromagnets. For Co $F^{2 \mathrm{D}}=2.0 \ldots 2.6,{ }^{8,10}$ for $(\mathrm{Ga}, \mathrm{Mn}) \mathrm{As} F^{2 \mathrm{D}}$ $=1.8 \ldots 2.6,{ }^{32,33}$ and for $\mathrm{Co} / \mathrm{Pt}$ multilayers $F^{2 \mathrm{D}}=2.5 .^{9} \mathrm{In} \mathrm{Fe}$ and $\mathrm{Ni}$ the parameters are comparable to the parameter observed in Co. ${ }^{10}$ We note that the effect of EEI is rather small in the quasi-two-dimensional film. The relative conductivity change $\Delta \sigma / \sigma$ from $1 \mathrm{~K}$ to $35 \mathrm{mK}$ is only $\sim 8 \times 10^{-4}$.

The magnetoconductance of the wire array, taken at 500 $\mathrm{mK}$, is shown in the inset of Fig. 3(b). Also in the wire array the AMR is visible but saturates already at $\approx 0.6 \mathrm{~T}$. This is due to the different shape anisotropy compared to an extended film. ${ }^{28}$ The conductivity decrease with respect to 20 $\mathrm{mK}$ is plotted in Fig. 3(b) for $B=6 \mathrm{~T}$ and zero field. Also in the wire array the conductivity decrease is independent of the applied magnetic field. Hence WL is absent also in the wire array. The temperature dependency of the conductivity decrease is rather different in the wire array and the Py-square. In the wire array $\Delta \sigma$ follows a $1 / \sqrt{T}$ dependency as it is expected for EEI in quasi-one-dimensional samples, ${ }^{29}$

$$
\Delta \sigma=-\frac{F^{1 \mathrm{D}}}{\pi A} \frac{e^{2}}{\hbar} \sqrt{\frac{\hbar D}{k_{B} T}},
$$

with the parameter $F^{1 \mathrm{D}}=(2-0.75 \tilde{F}) / \sqrt{2}$, the wire crosssection A, and the thermal diffusion length $L_{T}=\sqrt{\frac{\hbar D}{k_{B} T}}$. Using $D=4 \times 10^{-4} \mathrm{~m}^{2} / \mathrm{V} \mathrm{s} \quad$ (Ref. 18) and $\delta \sigma \sqrt{T}=-2.8$ $\times 10^{8}\left(e^{2} / h\right)(\sqrt{\mathrm{K}} / \mathrm{m})$ [see Fig. 3(b)] one obtains as parameter $F^{1 \mathrm{D}}=0.77$. Also the parameter $F^{1 \mathrm{D}}$ is in excellent agreement compared to the parameter obtained for other quasi-1D ferromagnets $\left[F^{1 \mathrm{D}}=0.83\right.$ in $\mathrm{Ni}$ (Ref. 11) and $F^{1 \mathrm{D}}$ $=0.72 \ldots 0.80$ in $(\mathrm{Ga}, \mathrm{Mn}) \mathrm{As}$ (Ref. 33)]. In the quasi-onedimensional wire array the effect of EEI becomes quite large compared to the quasi-two-dimensional film, as predicted by theory. ${ }^{29}$ In $1 \mathrm{D}$ the conductivity decreases by approximately $1.1 \%$ from $1 \mathrm{~K}$ to $35 \mathrm{mK}$. This is by a factor of 14 larger than the conductivity decrease in the quasi-two-dimensional film.

Although weak localization in ferromagnetic metals is strongly suppressed compared to nonmagnetic metals, the size of the conductivity correction due to electron-electron interaction is quite similar. For example the parameter $F^{2 \mathrm{D}}$ obtained in 2D AuPd is 2.1. ${ }^{34}$ In 1D AuPd and in 1D Au the parameters $F^{1 \mathrm{D}}$ are 1.36 (Ref. 34) and 0.93 (Ref. 35), respectively. This is in good agreement with the parameters observed here.

\section{SUMMARY}

We investigated quantum interference effects in mesoscopic permalloy films and wires at millikelvin temperatures. Our analysis of universal conductance fluctuations in single 
wires reveals a phase coherence length of $\sim 250 \mathrm{~nm}$ at 25 $\mathrm{mK}$. Compared to other ferromagnets this value is relatively large [in Co $L_{\phi}=30 \mathrm{~nm}$ at $30 \mathrm{mK}$ (Ref. 5) and in Ni $L_{\phi}$ $=80 \mathrm{~nm}$ at $30 \mathrm{mK}$ (Ref. 18)]. A possible explanation was given by Kasai et al. ${ }^{18}$ In ferromagnets the phase coherence length gets probably reduced with increasing magnetocrystalline anisotropy energy. The observed temperature dependency of the dephasing time $\propto T^{-1 / 2}$ is stronger than expected for Nyquist scattering in $1 \mathrm{D}$ systems $^{27}\left(\propto T^{-1 / 3}\right)$. A similar result was already obtained by Lee et $a l^{7}$ investigating TDUCF in permalloy. A microscopic mechanism for the $T^{-1 / 2}$ temperature dependence is still missing. Although the phase coherence length is relatively large, the conductance in 1D and 2D permalloy is not affected by weak localization. The decreasing conductance with decreasing temperature can be well described by EEI, as already shown for other ferromagnetic metals. ${ }^{8-11}$ The excellent agreement of the parameters $F^{2 \mathrm{D}}$ and $F^{1 \mathrm{D}}$ found in the ferromagnets investigated up to now underlines the universal character of EEI. The size of the WL correction is expected to be of the same order as EEI at zero field. ${ }^{29}$ Hence our data show that WL is strongly suppressed or even absence in permalloy. The absence of a Cooperon contribution in permalloy, the process leading to WL, was already proposed by Lee et $a l^{7}$ investigating $^{2}$ TDUCF. Although theory predicts the existence of WL in ferromagnets, ${ }^{30,31}$ it seems that the Cooperon contribution is suppressed in ferromagnets, independent of the value of the phase coherence length. The process which could lead to such a suppression is still unknown. Hence, further investigations, experimental as well as theoretical, are necessary to clarify the role of the internal magnetic induction on the phase-coherent transport in general and on the Cooperon in particular.

\section{ACKNOWLEDGMENTS}

This work was financially supported by the Deutsche Forschungsgemeinschaft (DFG) under Grant No. SFB 689. *daniel.neumaier@physik.uni-regensburg.de

${ }^{1}$ G. Bergmann, Phys. Rep. 107, 1 (1984).

${ }^{2}$ P. A. Lee, A. D. Stone, and H. Fukuyama, Phys. Rev. B 35, 1039 (1987).

${ }^{3}$ Y. Aharonov and D. Bohm, Phys. Rev. 115, 485 (1959).

${ }^{4}$ Y. Imry, Introduction to Mesoscopic Physics (Oxford University Press, Oxford, 1997).

${ }^{5}$ Y. G. Wei, X. Y. Liu, L. Y. Zhang, and D. Davidović, Phys. Rev. Lett. 96, 146803 (2006).

${ }^{6}$ S. Kasai, E. Saitoh, and H. Miyajima, Appl. Phys. Lett. 81, 316 (2002).

${ }^{7}$ S. Lee, A. Trionfi, and D. Natelson, Phys. Rev. B 70, 212407 (2004).

${ }^{8}$ M. Brands, C. Hassel, A. Carl, and G. Dumpich, Phys. Rev. B 74, 033406 (2006).

${ }^{9}$ M. Brands, A. Carl, and G. Dumpich, Ann. Phys. 14, 745 (2005).

${ }^{10}$ M. Brands, A. Carl, O. Posth, and G. Dumpich, Phys. Rev. B 72, 085457 (2005).

${ }^{11}$ T. Ono, Y. Ooka, S. Kasai, H. Miyajima, K. Mibu, and T. Shinjo, J. Magn. Magn. Mater. 226, 1831 (2001).

${ }^{12}$ H. Ohno, A. Shen, F. Matsukura, A. Oiwa, A. Endo, S. Katsumoto, and Y. Iye, Appl. Phys. Lett. 69, 363 (1996).

${ }^{13}$ D. Neumaier, K. Wagner, S. Geiszler, U. Wurstbauer, J. Sadowski, W. Wegscheider, and D. Weiss, Phys. Rev. Lett. 99, 116803 (2007).

${ }^{14}$ L. P. Rokhinson, Y. Lyanda-Geller, Z. Ge, S. Shen, X. Liu, M. Dobrowolska, and J. K. Furdyna, Phys. Rev. B 76, 161201(R) (2007).

${ }^{15}$ S. Washburn and R. A. Webb, Rep. Prog. Phys. 55, 1311 (1992).

${ }^{16}$ S. Datta, in Electronic Transport in Mesoscopic Systems, edited by A. L. Efros and M. Pollak (Elsevier, New York, 2002).

${ }^{17}$ J. J. Lin and J. P. Bird, J. Phys.: Condens. Matter 14, R501 (2002).

${ }^{18}$ S. Kasai, E. Saitoh, and H. Miyajima, J. Appl. Phys. 93, 8427
(2003).

${ }^{19}$ C. W. J. Beenakker and H. van Houten, Solid State Phys. 44, 1 (1991).

${ }^{20}$ T. R. McGuire and R. I. Potter, IEEE Trans. Magn. 11, 1018 (1975).

${ }^{21}$ K. Wagner, D. Neumaier, M. Reinwald, W. Wegscheider, and D. Weiss, Phys. Rev. Lett. 97, 056803 (2006).

${ }^{22}$ L. Vila, R. Giraud, L. Thevenard, A. Lemaître, F. Pierre, J. Dufouleur, D. Mailly, B. Barbara, and G. Faini, Phys. Rev. Lett. 98, 027204 (2007).

${ }^{23}$ V. Chandrasekhar, P. Santhanam, and D. E. Prober, Phys. Rev. B 42, 6823 (1990).

${ }^{24}$ F. Pierre, A. B. Gougam, A. Anthore, H. Pothier, D. Esteve, and N. O. Birge, Phys. Rev. B 68, 085413 (2003).

${ }^{25}$ D. Neumaier, K. Wagner, U. Wurstbauer, M. Reinwald, W. Wegscheider, and D. Weiss, N. J. Phys. 10, 055016 (2008).

${ }^{26}$ P. Dai, Y. Zhang, and M. P. Sarachik, Phys. Rev. B 46, 6724 (1992).

${ }^{27}$ B. L. Altshuler, A. G. Aronov, and D. E. Khmelnitsky, J. Phys. C 15, 7367 (1982).

${ }^{28}$ A. Hubert and R. Schäfer, Magnetic Domains (Springer, Berlin, 1998).

${ }^{29}$ P. A. Lee and T. V. Ramakrishnan, Rev. Mod. Phys. 57, 287 (1985).

${ }^{30}$ V. K. Dugaev, P. Bruno, and J. Barnaś, Phys. Rev. B 64, 144423 (2001).

${ }^{31}$ S. Sil, P. Entel, G. Dumpich, and M. Brands, Phys. Rev. B 72, 174401 (2005).

${ }^{32}$ H. Ohno and F. Matsukura, Solid State Commun. 117, 179 (2001).

${ }^{33}$ D. Neumaier, M. Schlapps, U. Wurstbauer, J. Sadowski, M. Reinwald, W. Wegscheider, and D. Weiss, Phys. Rev. B 77, 041306(R) (2008).

${ }^{34}$ J. J. Lin and N. Giordano, Phys. Rev. B 35, 545 (1987).

${ }^{35}$ W. D. Williams and N. Giordano, Phys. Rev. B 33, 8146 (1986). 